\title{
Optimised dynamic line scanning thermography for aircraft structures
}

\author{
by J. Peeters*, S. Verspeek*, S Sels, B. Bogaerts, G. Steenackers ${ }^{\star, \star *}$ \\ * University of Antwerp, Op3Mech Research Group, Groenenborgerlaan 171, B-2020 Antwerp, Belgium, \\ Jeroen.peeters2@uantwerpen.be; Simon.Verspeek@uantwerpen.be; Gunther.steenackers@uantwerpen.be \\ ${ }^{\star \star}$ Vrije Universiteit Brussel (VUB), Acoustics \& Vibration Research Group (AVRG), B-1000 Brussels, Belgium
}

\begin{abstract}
In-line inspection of advanced components, remains a challenging task in industry. A methodology is discussed which uses numerical simulations to automatically determine the best set of experimental parameters to inspect the structure on defects using active thermography. The inspection is performed using an industrial conveyor belt or a robotic arm optimised using a numerical model of the defected test sample. During the path planning, the directional emissivity is considered for the complex surface and an optimal experimental setup is found. The results show that in-line quality evaluation of complex shaped structures is improved with a minimal amount of inspection time.
\end{abstract}

\section{Introduction}

The use of fibre reinforced composites, such as carbon fibre reinforced plastics (CFRP) is growing each year. They already showed their usability in aerospace structures for a few decades, but are starting to enter general consumer markets as well. With the increased use of those materials, there is a need for efficient quality assurance techniques, economical and effective repair techniques and methods to predict the short- and long-term behaviour of the composite structures under a variety of loading and environmental conditions [1].

Active thermography is a widely used technology to inspect large CFRP components for flaws in a fast way [2]. To receive accurate results on large samples, the thermal camera has to be repositioned over the structure in a way each surface is inspected with sufficient accuracy with an equal heat excitation and time resolution considering directional emissivity [3]. To perform these sequential experiments in a robust, repetitive way, multiple techniques are developed which mount a thermal camera and excitation source or the test sample to a translation unit to control the relative position and speed of the inspection unit versus the inspected structure [4,5]. Unfortunately, those systems are complex, expensive and are restricted to safety regulations. To further decrease inspection time and costs for large flat structures, a dynamic line scanning principle is described in recent literature $[6,7]$. This technology could be used for continuously formed components and is especially useful when the width is rather limited.

Automatic scanning using a robotic arm or line translator such as a conveyor belt, is seen as an interesting way for Non-Destructive Testing (NDT) of large and complex shaped components [8]. The programming of the inspection path, speed, excitation power/ duration, excitation distance and acquisition rate is a difficult task which is highly dependent on the inspected sample geometry, material properties and interested depth resolution $[8,9]$. This parameter optimisation is mostly done manually by a highly qualified inspector using experience and trial and error, which is a time-consuming task for complex structures as the heat deposition and scanning speed should match the heat diffusion in the material [10]. Especially when using industrial robots with multiple degrees of freedom (DOFs), this could become a cumbersome task. The goal of this paper is to inspect a carbon fibre reinforced plastic (CFRP) structure and inspect for anomalies such as missing foam and delaminations using a continuously scanning system on an industrial conveyor belt. The limitations of a conveyor belt are found in the biasing movement vibrations and non-linear movement which might occur.

\section{Methodology}

A numerical simulation using Finite Element Analysis and robotic simulation software is used to optimise the complex parameter set of the robotic thermography set-up by correlating the numerical results with experimental thermography data. In addition, path planning tools are used in order to verify the visibility of the full structure in combination with the directional emissivity of the test sample. This results in optimal trajectory planning for the experimental recording.

\subsection{Experimental measurements}

The measurements are performed on a CFRP test panel of a Fokker Horizontal Tail Plane (HTP) which is manually laminated with pre-preg unidirectional fibre plies, vacuum bagged and cured using a two stage pressurised autoclave curing at 5.86 bar and $120^{\circ} \mathrm{C}$ under vacuum for $65 \mathrm{~min}$ and 6.9 bar and $177^{\circ} \mathrm{C}$ vented for 130 min. The ply stacking of the stringer caps which are the focus for this research is [OF, OUD, 45F, OUD]s with a Rohacell foam core.

The methodology uses a conveyor belt with a controllable speed between $1 \mathrm{~mm} / \mathrm{min}$ and $1000 \mathrm{~mm} / \mathrm{min}$, a Xenics Gobi micro-bolometric thermal camera $(7-14 \mu \mathrm{m}, 640 \times 480$ pixels) and an halogen optical excitation line source of max 
$5000 \mathrm{~W} / \mathrm{m}$, used at a power consumption of $40 \%$. The camera records at $8 \mathrm{~Hz}$ when the conveyor belt is set to a speed of $10 \mathrm{~mm} / \mathrm{sec}$, this corresponds to a cooling down window of $60 \mathrm{sec}$. The set-up is shown in Fig. 1 . The measurements are compared with long pulse thermal measurements acquired with a FLIR X6540sc cooled MWIR camera (3-5 $\mu \mathrm{m}, 640 \times 512$ pixels) and halogen optical excitation of $30 \mathrm{sec}$ of $1000 \mathrm{~W}$ at a distance of $1 \mathrm{~m}$ in vertical position. To compare the measurements with the conveyor belt measurements, a cooling down process is measured for 60sec at a frame rate of $30 \mathrm{~Hz}$. In the last step, the conveyor belt measurements are repeated with an industrial 6 axis robot arm (Kuka KR16) following the resulting paths of the optimisation and visibility study described in Section 2.4. The measurements are performed with the same camera as the conveyor belt measurements and with a similar heating source.
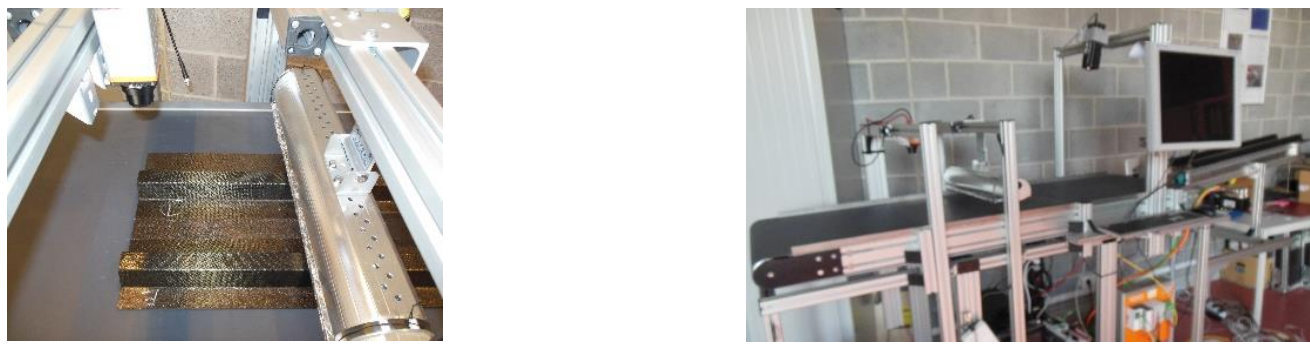

Fig. 1. Dynamic line scan setup using a conveyor belt.
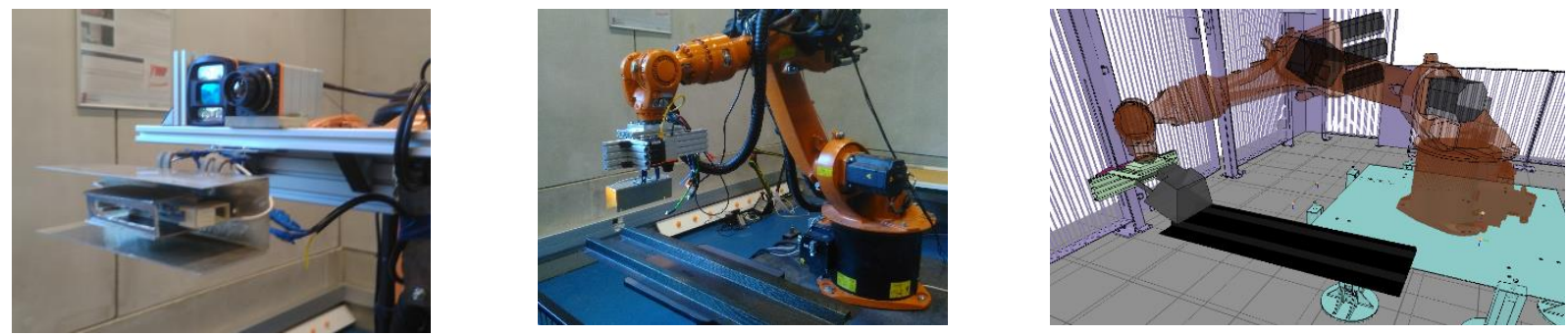

Fig. 2. Dynamic line scan setup using an industrial robot arm and its corresponding simulation environment.

\subsection{Delamination creation \& simulation}

Standard destructive analysis is performed in order to create realistic delaminations at controlled positions in the structure. A compression with a stamp of $5 \mathrm{~mm}$ diameter is used in order to create the delaminations with an impact energy of 1, 3 and $4 \mathrm{~J}$ respectively. In addition, $60 \mathrm{~mm}$ of Rohacell foam is removed in one of the stingers, before the delaminations were created. A schematic representation of the defect positions is shown in Fig. 3. An impact energy profile is given in Fig. 4. Only the defect of $4 \mathrm{~J}$ is visible by the naked eye due to external damage of the laminate. It is supposed to be that the impact of $1 \mathrm{~J}$ would be difficult to verify using active thermography and the $3 \mathrm{~J}$ and $4 \mathrm{~J}$ impact would create some significant delaminations in the structure [11]. By the creation of all defects, some fibre cracking appeared.

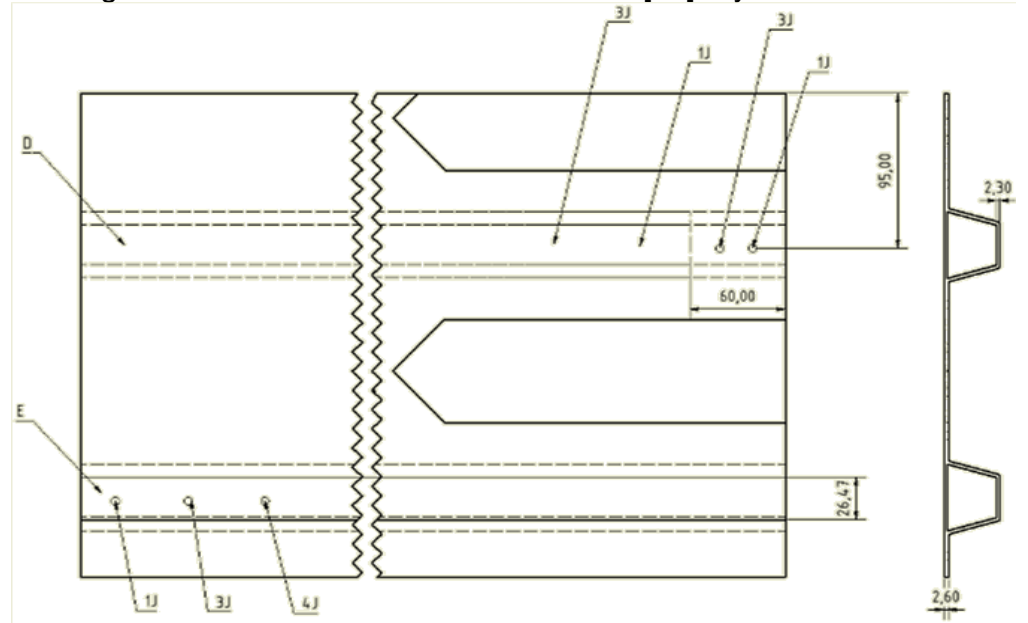

Fig. 3. Test sample defect locations.

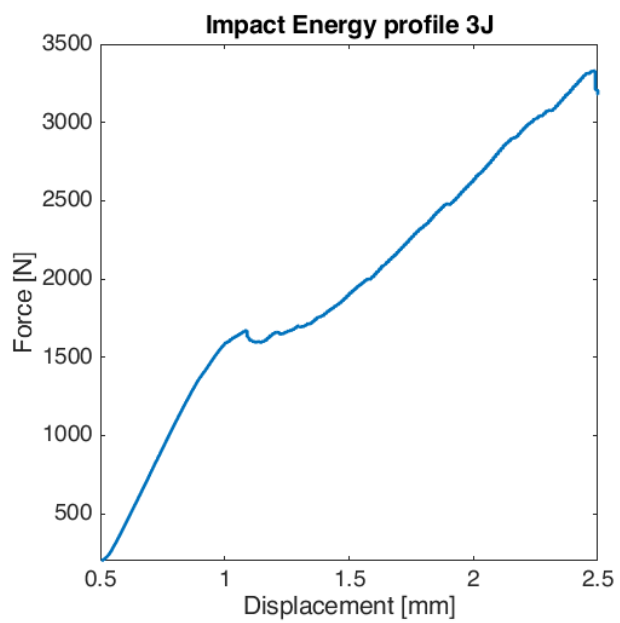

Fig. 4. Impact Energy profile 3J. 


\subsection{Numerical simulations}

The numerical model is developed in Siemens Simcenter 12 combining transient heat transfer with multibody dynamics.It is performed in combination with the Samcef nonlinear structural solver for simulating the delaminated zones resulting from the destructive impact test. The anisotropic thermal conductivity through the CFRP layers and its influence on the global thermal diffusivity is considered, as further discussed in [9].

\subsubsection{Structural non-linear simulation}

Initially, a structural non-linear model of the multiple plies, stacked as described in Section 2.1, is created. The foam and CFRP are connected using a flexible to flexible connection property without friction, including the glue properties of Epoxy resin. This results in an interaction between the foam and the CFRP laminate locally at the pressure point. The delaminations are reconstructed in the numerical model, using the boundary constrains of the delamination creation test. The bottom of the hood stringer is constrained using a rigid contact surface. The force is approximated with a point force which is connected with a rigid link to the different elements of the top layer of the hood stringer with a similar contact surface as the real stamp ( $5 \mathrm{~mm}$ diameter). All boundary conditions are shown in Fig. 5 . The model is solved using the nonlinear Samcef Mecano solver of Samtech The displacement output, shown in Fig. 6, containing the delamination area, is used as input mesh to create a new, damaged geometry. This new geometry is further used for the thermal simulations.

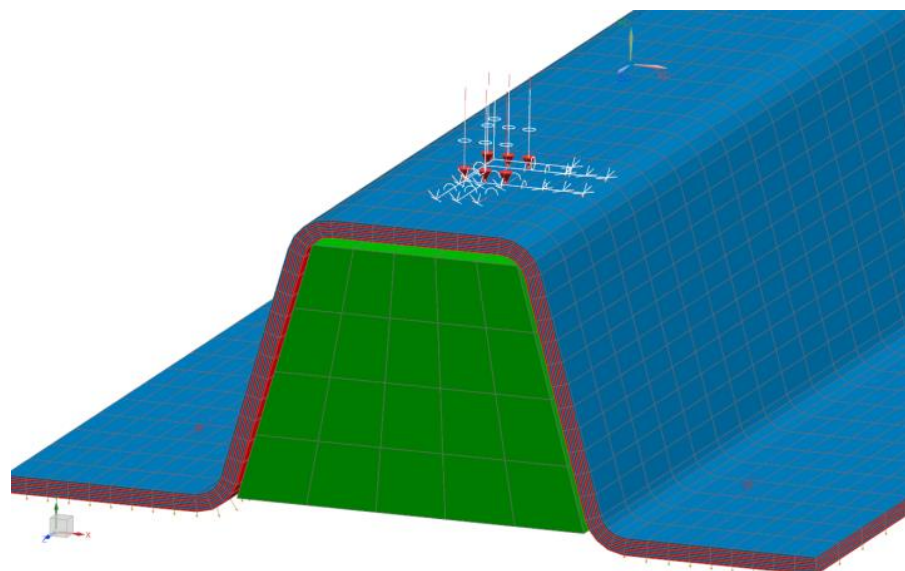

Fig. 5. Structural model setup, rohacell (green), Force vector (Red), DOF's (White)

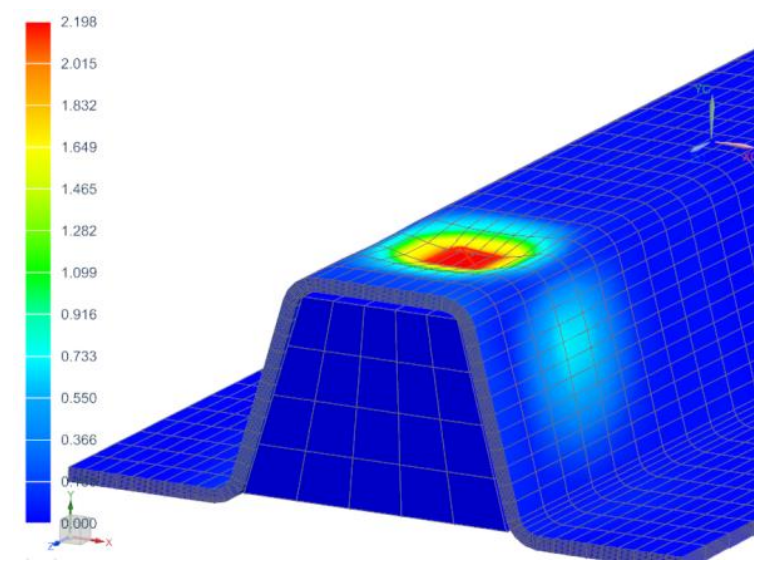

Fig. 6. Simulated delamination at maximal deflection

\subsubsection{Thermal simulation}

The thermal model is built using the NX thermal solver. The thermal material properties are found in the CES material database [12] for unidirectional pre-preg CFRP with Epoxy matrix material and Rohacell foam. The heat source of $2000 \mathrm{~W} / \mathrm{m}$ is approximated with a moving $2 \mathrm{D}$ mesh at a constant speed. The moving mesh contains a boundary heat source which emits Gaussian diffuse radiation. The view factors are computed using a Monte Carlo simulation in order to evaluate for each position of the moving heat source, the intensity received by the top layer mesh elements of the test sample. As the heat source moves over the test sample, the view factors variate over time.

Convective heat transfer is computed automatic dependent for natural convection, considering the inclination angle of the sector of the test sample. Radiation is considered between the heat source and the test sample primarily using the deterministic Monte Carlo view factor computation of the heat source. Additionally a radiative contact is used in order to simulate the radiation inside the cavity where the foam is missing. A thermal conductivity contact is used between the Rohacell and CFRP in order to simulate the disrupted contact of the epoxy glue. The glue thickness is approximated by a thermal conductance gap with a reduced thermal conductivity equal to the thermal conductivity of the epoxy resin, found to be $0.188 \mathrm{~W} / \mathrm{m} \cdot \mathrm{K}[12]$.

The governing differential equation is a combined heat transfer equation with conduction, convective cooling and radiation of an external boundary heat source, formulated in Eq.(1), Eq.(2) and Eq.(3) where $\rho$ is the density, $C_{p}$ is the heat capacity at constant pressure, $\mathrm{T}$ is the absolute surface temperature, $\mathrm{K}$ is the thermal conductivity, $\mathrm{t}$ is the time, $\mathrm{Q}(\mathrm{t})$ is the time dependent heat source, $h$ is the coefficient of convection and $\sigma$ is the constant of Stefan-Boltzmann $[13,14]$.

$$
\begin{aligned}
\rho C_{p} \frac{\partial T}{\partial t}+\nabla \cdot(-\kappa \nabla T) & =Q \text { with } T(x, y, z, 0)=T_{\infty}=292.88[K] \\
\vec{n}(\mathrm{k} \nabla \mathrm{T}) & =\mathrm{h}\left(T_{\infty}-\mathrm{T}\right)
\end{aligned}
$$




$$
\vec{n}(\mathrm{k} \nabla \mathrm{T})=\varepsilon \cdot \sigma \cdot\left(T_{\infty}^{4}-T^{4}\right)
$$

A few assumptions are made:

- The air velocity is assumed to be constant and inverse to the measurement direction due to the movement.

- The lay-up of the CFRP laminate is considered quasi-isotropic with structured fibre angles in a woven pattern.

- The test sample is opaque and behaves like an ideal grey body as a result of the mate epoxy surface.

The developed model is shown in Fig. 7 with the moving boundary heat source in yellow and the test sample in green. It should be remarked that in the simulation model the full width of the sample is heated and evaluated at once. In reality, especially in a full continuous robotic inspection, historical preheating could bias the inspection results. A simulation result is shown in Fig. 8, where the missing foam appears as a solid hot spot after heating and the delamination results in a lower conductance which results in smaller hot spot.

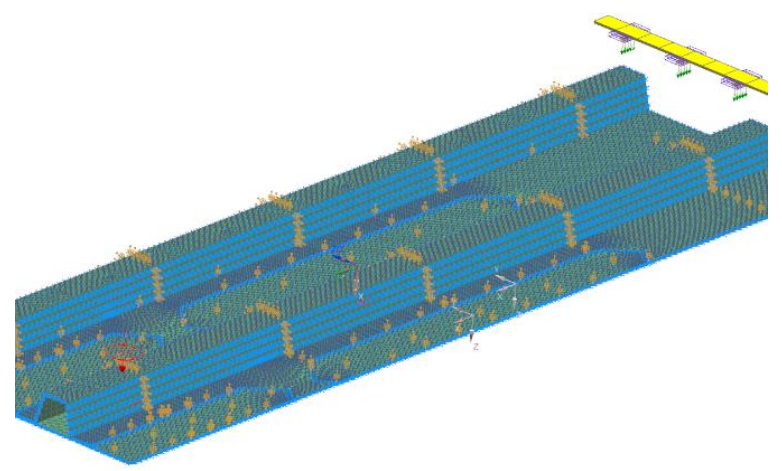

Fig. 7. Thermal model setup, with moving mesh boundary heat source (yellow)

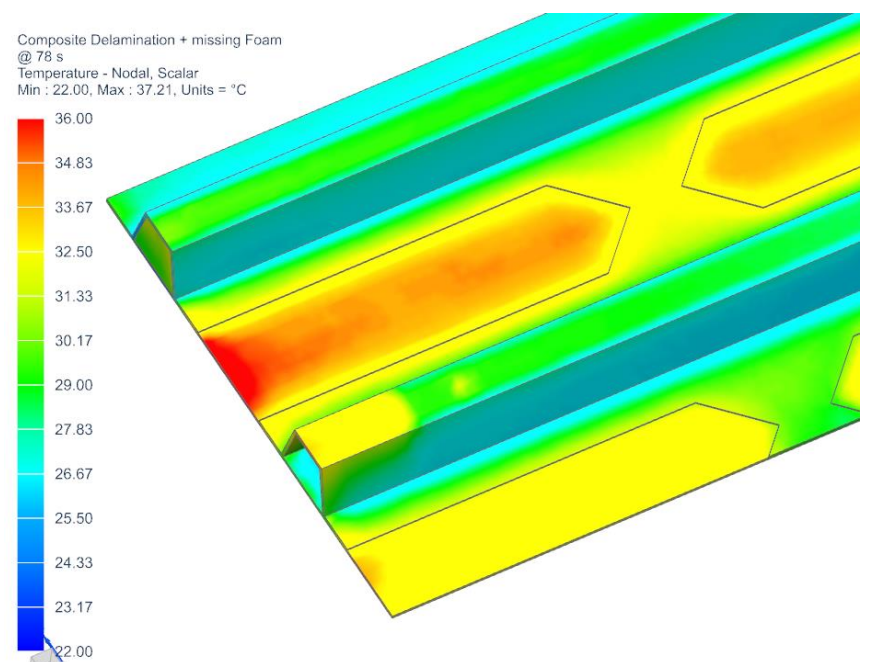

Fig. 8. Thermal result of cooling discrepancy of missing foam and delamination

\subsection{Optimisation \& path planning}

The path planning is dependent on the directional emissivity of the structure. To automatically determine an efficient scanning trajectory we use a greedy 'generate and test procedure' similar to [16]. We first compute a set of candidate viewpoints. For every candidate viewpoint of the thermal camera we calculate the directional emissivity in every mesh node of the test sample surface, which is dependent on the scanning angle. The directional emissivity is defined using Eq. (4), where $p_{1}$ and $p_{2}$ are fitting parameters, found using a numerical model updating according to [3] as, respectively, $\sqrt{1.6}$ and $1.115 \pi$. The maximal emissivity is set equal to the normal emissivity measured experimentally. We evaluate the quality of the mean directional emissivity over the mesh nodes to preselect configurations for the thermal simulation. This set of best value is stored as current measurement state for each node. This step is then repeated until stopping criteria are met. These stopping criteria can be 'maximum coverage reached' or 'maximum number of viewpoints reached'.

$$
\varepsilon(\theta)=\max \left(\cos \left(p_{1} \theta+p_{2} \cdot \pi\right), 0\right)
$$

The constraints of the dynamic line scan setup are of great importance to the final scanning trajectory. To simulate these effects we use robotic simulation software [17]. In this software we can model our complete measurement setup. This software will take care of robot specific complex path planning, limitations and collision checking.

The quality of the final path is highly dependent on the positioning of the test sample due to collisions and directional emissivity. Therefore we efficiently sample the configuration space of the structure and select the position which delivers the best scanning trajectory. Those optimal scanning trajectories are used as input data for the thermal simulations, together with some initial experimental measurement data as reference set. 


\section{Results \& Discussion}

Within the results section, we show a comparison between a standard step heated active thermography measurement using a high-end cooled thermal camera and at first a continuous DLST system with a conveyor belt and secondly optimised robotic inspection using the path planning to evaluate the local material thickness using low-cost measurement equipment. A comparison will be made on the accuracy of the evaluation of the delaminations and large part of missing Rohacell foam. The time consumption and accuracy of the optimised inspection using the conveyor belt will be discussed and compared with the fixed position inspection of the step heating measurements and the robotic line scan measurements. The numerical model gave the minimum excitation power (40\%) and optimal translation speed of $10 \mathrm{~mm} / \mathrm{sec}$ for the specified test sample in order to distinguish both delaminations and missing part of Rohacell foam. The visibility study provided the optimal camera distance and spatial camera positioning in order to minimize the path length and provide an optimal emissivity for the full test sample.

\subsection{Step heating experiment (benchmark)}

Only the cooling phase of the long duration pulse thermography measurement is considered in order to compare with the DLST measurements. The measurements are performed separately for hood stringer D and E as shown in Figure 2. The results are post-processed using Principal Component Thermography (PCT) in order to improve the signal to noise ratio. In Fig. 9, the part where the Rohacell foam is missing in hood stringer $D$ is clearly seen due to a reduced conductance. In Fig. 10, the same hood stringer is shown in the sixth bin of the PCT analysis. The resulting delamination of the impact of $3 \mathrm{~J}$ could be seen, especially above the part where the Rohacell supports the CFRP hood stringer. Due to the absence of the Rohacell below the left zone, the delamination is smaller of an equal amount of impact energy. This could be caused due to a larger deflection due to the missing support of the Rohacell foam. It appears that the lower impacts of $1 \mathrm{~J}$ did not caused any delamination.

In hood stringer E, shown in Fig 11, it can be seen that the impact of $4 \mathrm{~J}$, made a large delamination surrounding the hole in the upper CFRP layers. In addition, the impact at $3 \mathrm{~J}$ could be seen due to a smaller delamination, which vaguely appears. The impact of $1 \mathrm{~J}$ could not be seen in this measurement as well. This strengthens the assumption that an impact of $1 \mathrm{~J}$ was insufficient to cause internal damage.

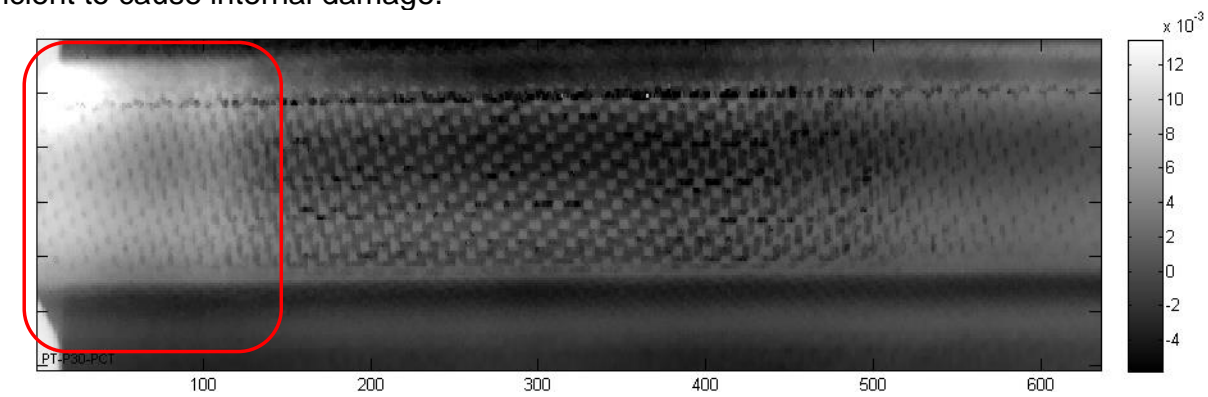

Fig. 9: Missing foam section of hood stringer D appears relatively fast and pronounced in the second PCT bin.

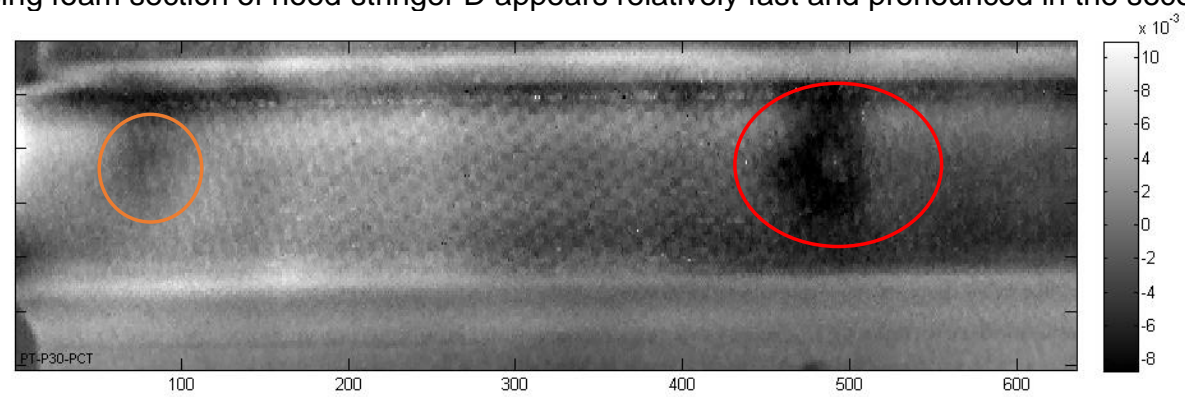

Fig. 10: Only the two larger impacts of 3J appear as a delamination on the hood stringer D in PCT bin 6.

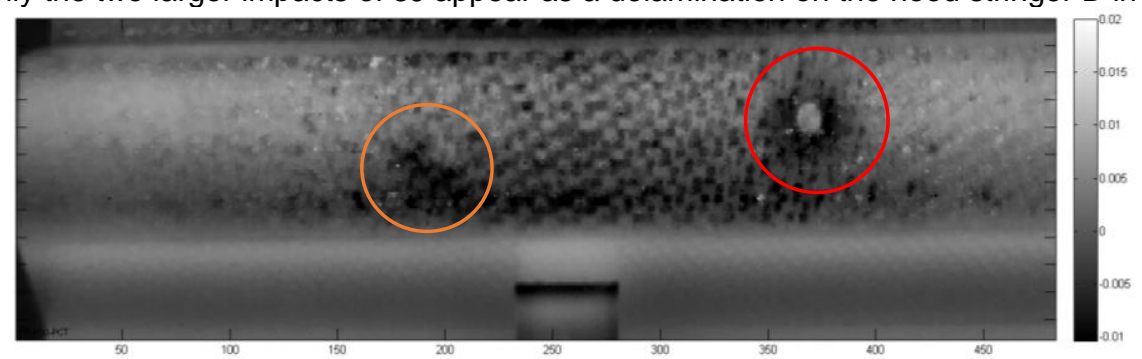

Fig. 11: Delaminations on hood stringer E, 


\subsection{Linear line scan using a conveyor belt}

The results in Fig. 12 - 14 show that using a conveyor belt type of linear translator in combination with a less accurate microbolometric camera, could only show the missing foam section. Two different post-processing techniques are used with similar results, the correlation over the full temporal range of 480 images, respectively $60 \mathrm{sec}(10 \mathrm{~mm} / \mathrm{sec})$ or $160 \mathrm{sec}(5 \mathrm{~mm} / \mathrm{sec})$ of cooling. On Fig. 13 , it is clearly seen that due to the movement of the conveyor belt, there is a tradeoff between elevated temperature due to the missing foam and an increased convective cooling of the remaining CFRP at the front side. This becomes more highlighted when the speed is slowed down to improve thermal resolution (Fig. 14) and the cavity receives more time to cool down. No delaminations could be distinguished and irregular movement might blur the results significantly when the speed is reduced. The movement blur is caused by the velocity error and vibrations of the conveyor belt.

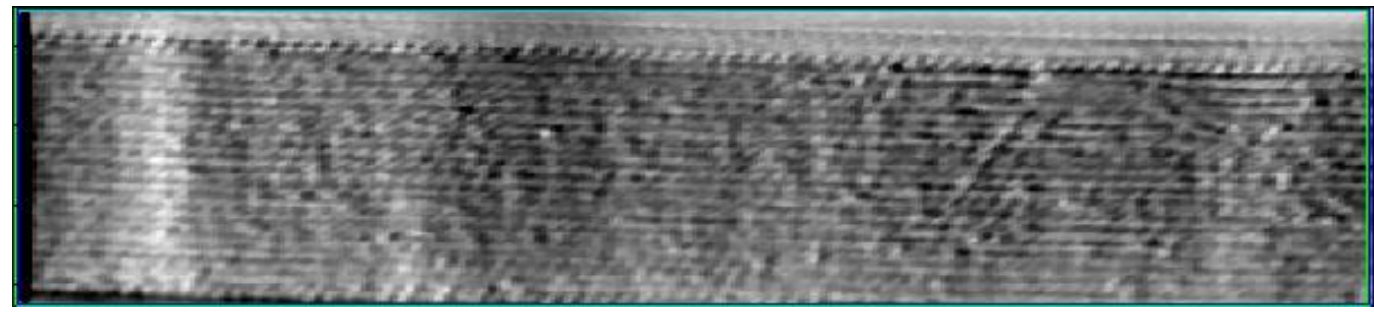

Fig. 12: Stringer D, 2000W/m power, translation speed of $10 \mathrm{~mm} / \mathrm{sec}, 8 \mathrm{~Hz}, \mathrm{PCT}$ bin 2

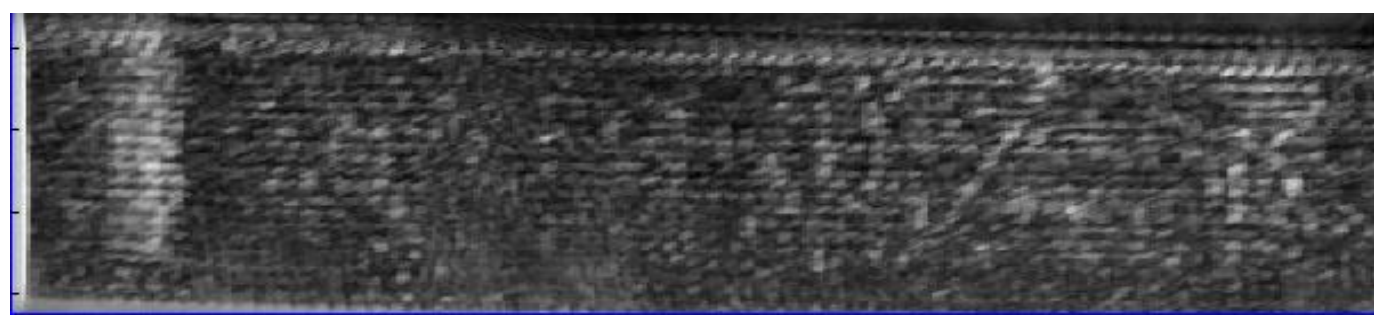

Fig. 13: Stringer D, 2000W/m power, translation speed of $10 \mathrm{~mm} / \mathrm{sec}, 8 \mathrm{~Hz}$, Correlation

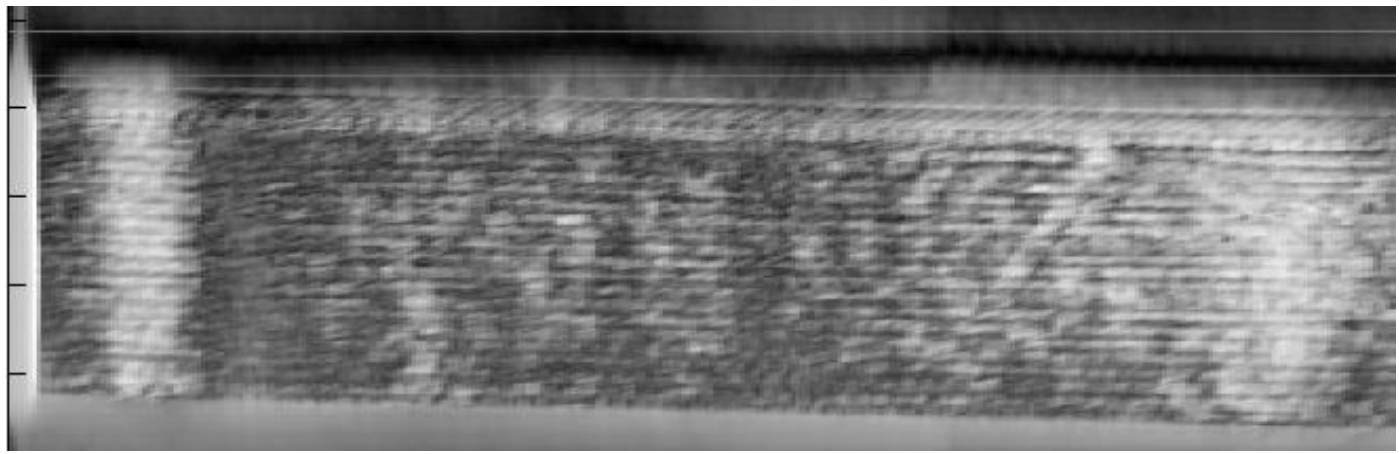

Fig. 14: Stringer D, 2000W/m power, translation speed of $5 \mathrm{~mm} / \mathrm{sec}, 3 \mathrm{~Hz}$, Correlation

\subsection{Dynamic line scan using an optimal path and robotic arm}

The results of the robotic paths is shown in Fig 15-19. As expected, the optimal paths are followed more accurate as by using the conveyor belt which improves the spatial contrast. As shown in Fig. 15 and 18, both delaminations in stringer $E$ and missing foam in stringer $D$ can be distinguished, vaguely at the optimal inspection parameters. When slowing down the inspection path, all defects appear even more promising at the same locations. In addition, as shown in Fig. 17 two anomalies can be seen at the positions of the delaminations of stringer D, on top of the missing foam, similar as in Fig. 10. Although it appears as the delamination is rather rectangular distributed. For this reason a high uncertainty remains with respect of the detection of those delaminations. 


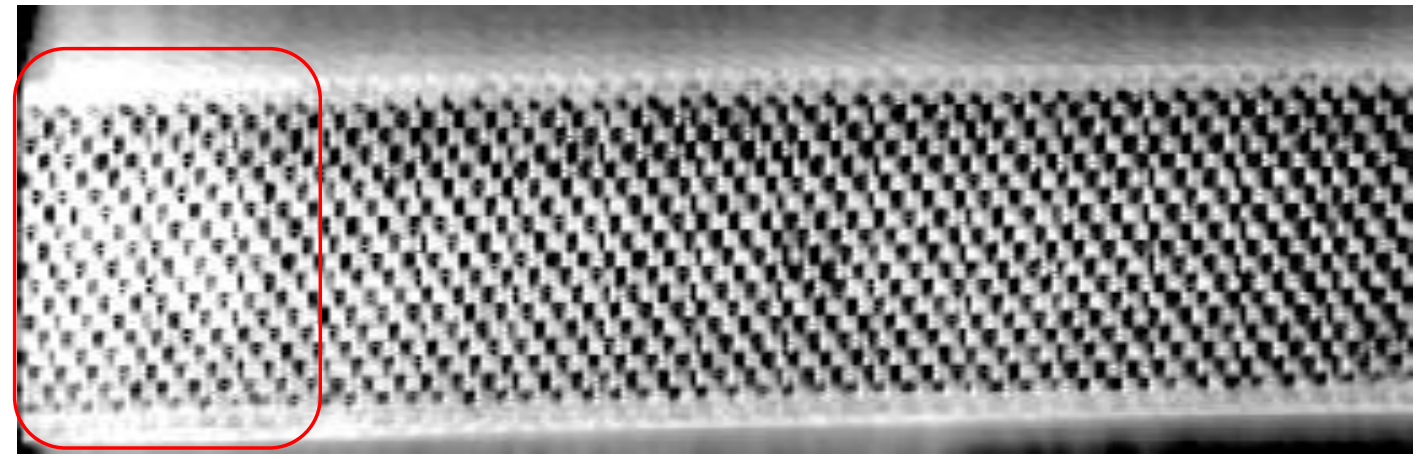

Fig. 15: Stringer D, 2000W/m power, translation speed of $10 \mathrm{~mm} / \mathrm{sec}, 8 \mathrm{~Hz}, \mathrm{PCT}$ bin 2

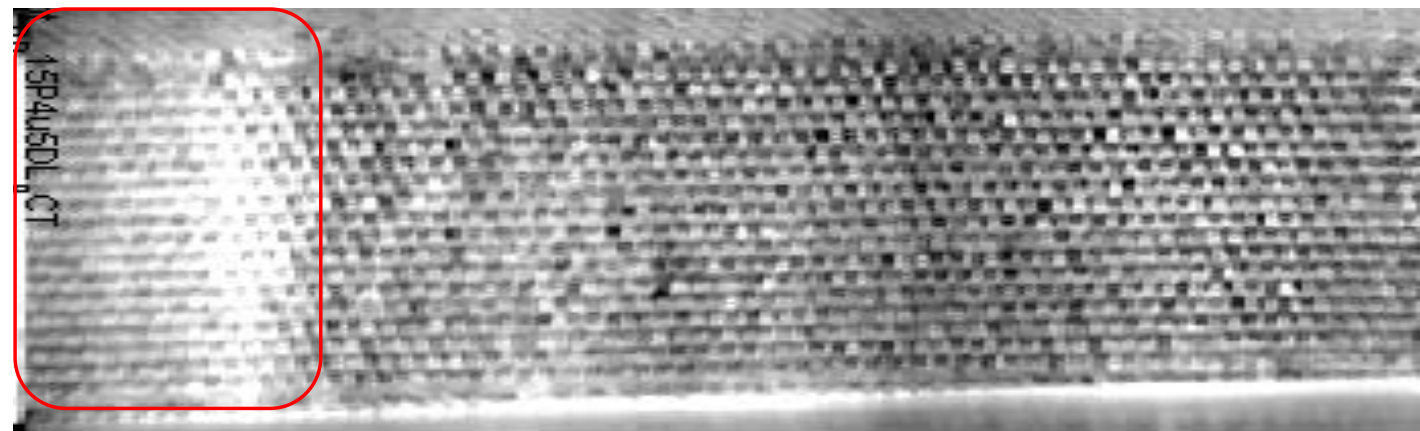

Fig. 16: Stringer D, 2000W/m power, translation speed of $5 \mathrm{~mm} / \mathrm{sec}, 3 \mathrm{~Hz}$, PCT bin 2

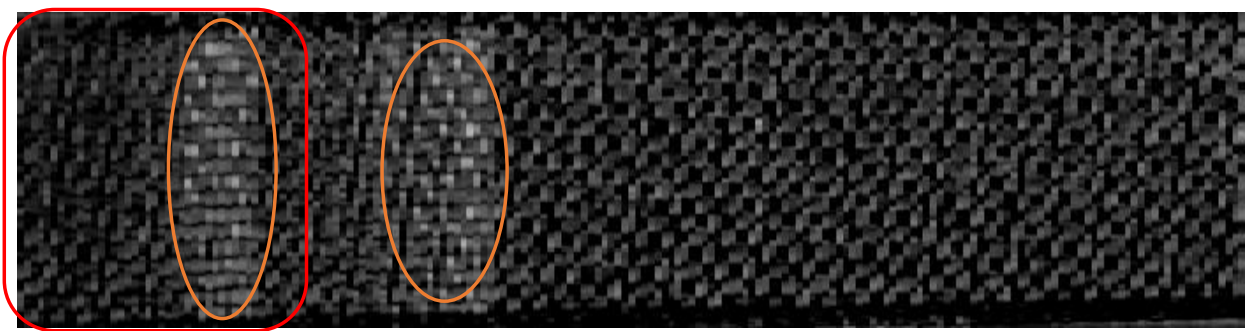

Fig. 17: Stringer D, 2000W/m power, translation speed of $5 \mathrm{~mm} / \mathrm{sec}, 3 \mathrm{~Hz}$, Correlation

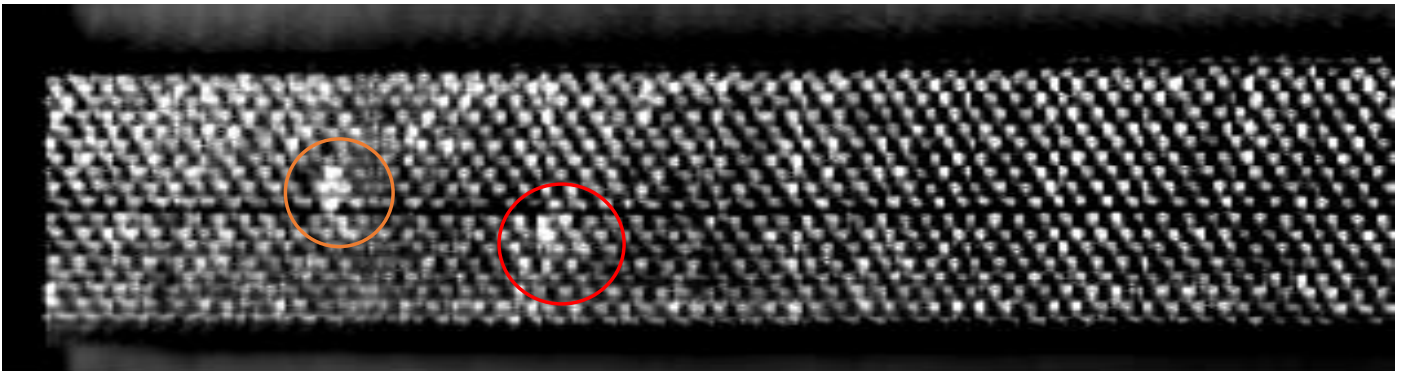

Fig. 18: Stringer E, 3000W/m power, translation speed of $10 \mathrm{~mm} / \mathrm{sec}, 8 \mathrm{~Hz}, \mathrm{PCT}$ bin 2

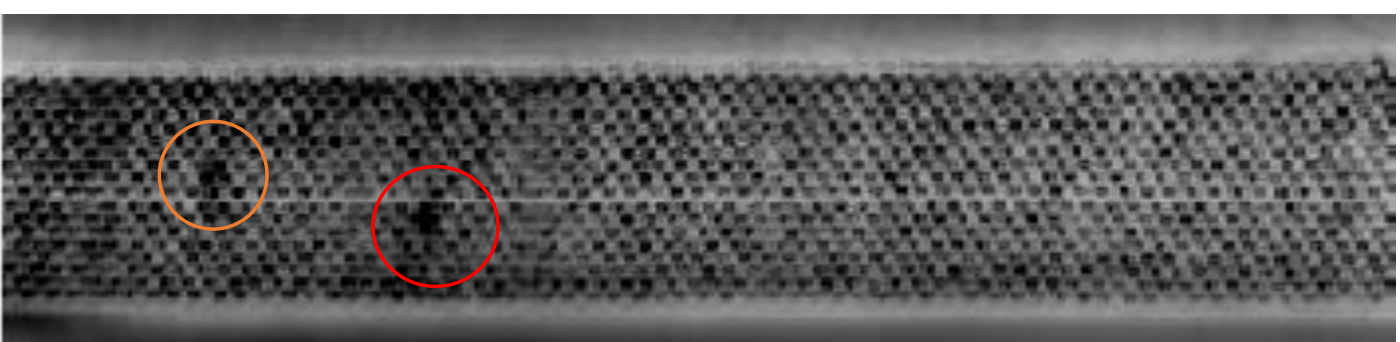

Fig. 19: Stringer E, 2000W/m power, translation speed of $5 \mathrm{~mm} / \mathrm{sec}, 3 \mathrm{~Hz}, \mathrm{PCT}$ bin 2 


\section{Conclusion and broader perspective}

We can conclude that the modification from academic standard tests such as long step thermography to a more industrial automated scale causes some difficulties. Especially for longer test samples, where continuous scanning techniques are needed. With the conveyor belt measurements, it is shown that some significant vibrations occur, which causes motion blur. In addition a full inspection of the test sample requires multiple cameras or a reduced spatial resolution. Especially when the translation speed is reduced in order to evaluate thicker test samples, the noise due to vibration increases. Additionally, industrial applications in standard commercial sectors require low budget alternatives where a cooled thermal camera is mostly not an option. It is shown that there are some alternatives, although movement vibrations could alter the accuracy especially at very low velocities. Although, due to the rolling shutter principle, those cameras do have a motion blur as well at higher inspection velocities. This could conclude that only a certain range of applications could benefit from the low budget microbolometric thermal cameras in combination with a conveyor belt. This could only become feasible for shallow evaluation of small test samples with medium to low conductive material properties. Within this work, it is shown that the implementation of an optimisation process using thermal-structural numerical model updating with advanced visibility analysis and robotic path planning might significantly improve the accuracy of automatic dynamic line scan thermography without the need of excessive calibration. It is shown that qualitative thickness evaluation and integrity control of complex shaped structures is feasible and a distinction can be made between the different defect types. In addition, future potential can be found in the link between visibility study and numerical model in order to consider preheating in an effective way similar to the directional emissivity. Future research is needed in order to compensate for the discontinuous movement in order to use the technique accurately on an industrial conveyor belt.

\section{Acknowledgment}

This research has been funded by the University of Antwerp and the IWT-VLAIO by the support to the TETRA project "Smart Integration of Numerical modelling and Thermal inspection (SINT)." The authors would like to end with a special thanks to the Op3Mech research team for the support on the preliminary measurements of this research and a special thanks to Boris Bogaerts and Seppe Sels for the assistance with the robotic measurements and Jean-Pierre Smet for his assistance during the destructive tests. In addition we would like to thank Sabca Limburg for providing the test sample.

\section{REFERENCES}

[1] A. Maier, R. Schmidt, B. Oswald-Tranta, R. Schledjewski, Non-destructive thermography analysis of impact damage on large-scale CFRP automotive parts, Materials (Basel). 7 (2014) 413-429. doi:10.3390/ma7010413.

[2] X. Maldague, Theory and practice of infrared thermography for nondestructive testing, Wiley, New York, 2001.

[3] J. Peeters, B. Ribbens, J.J.J. Dirckx, G. Steenackers, Determining directional emissivity: Numerical estimation and experimental validation by using infrared thermography, Infrared Phys. Technol. 77 (2016) 344-350. doi:10.1016/j.infrared.2016.06.016.

[4] K.E. Cramer, W.P. Winfree, Thermographic detection and quantitative characterization of corrosion by application of thermal line source, Proc. SPIE - Int. Soc. Opt. Eng. 3361 (1998) 291-300. doi:10.1117/12.304740.

[5] D.F. Woolard, K.E. Cramer, The thermal photocopier: a new concept for thermal NDT, in: SPIE, 2004 : p. 366. doi:10.1117/12.541881.

[6] H. Zhang, S. Sfarra, K. Saluja, J. Peeters, J. Fleuret, Y. Duan, H. Fernandes, N. Avdelidis, C. Ibarra-Castanedo, $X$. Maldague, Non-destructive Investigation of Paintings on Canvas by Continuous Wave Terahertz Imaging and Flash Thermography, J. Nondestruct. Eval. 36 (2017) 34. doi:10.1007/s10921-017-0414-8.

[7] F. Khodayar, F. Lopez, C. Ibarra-Castanedo, X. Maldague, Optimization of the Inspection of Large Composite Materials Using Robotized Line Scan Thermography, J. Nondestruct. Eval. 36 (2017). doi:10.1007/s10921-0170412-x.

[8] C. Ibarra-Castanedo, P. Servais, A. Ziadi, M. Klein, X. Maldague, RITA - Robotized Inspection by Thermography and Advanced processing for the inspection of aeronautical components camera heating source actuator camera heating, in: QIRT2014 Conférence, 2014: pp. 1-8. doi:http://dx.doi.org/10.21611/qirt.2014.164.

[9] J. Peeters, C. Ibarra-Castanedo, S. Sfarra, X. Maldague, J.J.J. Dirckx, G. Steenackers, Robust Quantitative Depth Estimation on CFRP samples using active thermography inspection and numerical simulation updating, NDT E Int. (2017). doi:10.1016/j.ndteint.2017.02.003.

[10] O. Ley, V. Godinez-Azcuaga, Line Scanning Thermography and its Application Inspecting Aerospace Composites, in: 5th Int. Symp. NDT Aerosp., 2013: pp. 13-15.

[11] R. Usamentiaga, C. Ibarra-Castanedo, M. Klein, X. Maldague, J. Peeters, A. Sanchez-Beato, Nondestructive Evaluation of Carbon Fiber Bicycle Frames Using Infrared Thermography, Sensors. 17 (2017) 1-16. doi:10.3390/s17112679.

[12] Granta Design Limited, CES Edupack 2013, (2013).

[13] A.J. Chapman, Heat transfer, 4th ed., Macmillan, New York, 1984. 
[14] B. Stotter, K.H. Gresslehner, G. Mayr, G. Hendorfer, J. Sekelja, Quantitative application of pulse phase thermography to determine material parameters, in: Quant. Infrared Thermogr., Bordeaux, 2014: p. 10. doi:http://dx.doi.org/10.21611/qirt.2014.074.

[15] J. Peeters, G. Arroud, B. Ribbens, J.J.J. Dirckx, G. Steenackers, Updating a finite element model to the real experimental setup by thermographic measurements and adaptive regression optimization, Mech. Syst. Signal Process. 64-65 (2015) 428-440. doi:10.1016/j.ymssp.2015.04.010

[16] W.R. Scott, Model-based view planning, Mach. Vis. Appl. 20 (2009) 47-69. doi:10.1007/s00138-007-0110-2.

[17] E. Rohmer, S.P.N. Singh, M. Freese, V-REP: A versatile and scalable robot simulation framework, in: 2013 IEEE/RSJ Int. Conf. Intell. Robot. Syst., IEEE, 2013: pp. 1321-1326. doi:10.1109/IROS.2013.6696520. 